\title{
Chromosome-scale genome assembly of Cucumis hystrix - a wild species interspecifically cross-compatible with cultivated cucumber
}

\author{
Xiaodong Qin', Zhonghua Zhang 2,3, Qunfeng Lou', Lei Xia', Ji Li', Mengxue Li', Junguo Zhou ${ }^{4}$, Xiaokun Zhao', \\ Yuanchao X $\mathrm{u}^{3}$, Qing $\mathrm{Li}^{3}$, Shuqiong Yang ${ }^{1}$, Xiaqing Yu ${ }^{1}{ }^{1}$, Chunyan Cheng ${ }^{1}$, Sanwen Huang ${ }^{5}$ and Jinfeng Chen ${ }^{1}$
}

\begin{abstract}
Cucumis hystrix Chakr. $(2 n=2 x=24)$ is a wild species that can hybridize with cultivated cucumber (C. sativus L., $2 n=$ $2 x=14$ ), a globally important vegetable crop. However, cucumber breeding is hindered by its narrow genetic base. Therefore, introgression from C. hystrix has been anticipated to bring a breakthrough in cucumber improvement. Here, we report the chromosome-scale assembly of C. hystrix genome (289 Mb). Scaffold N50 reached $14.1 \mathrm{Mb}$. Over 90\% of the sequences were anchored onto 12 chromosomes. A total of 23,864 genes were annotated using a hybrid method. Further, we conducted a comprehensive comparative genomic analysis of cucumber, C. hystrix, and melon (C. melo L., $2 n=2 x=24$ ). Whole-genome comparisons revealed that $C$. hystrix is phylogenetically closer to cucumber than to melon, providing a molecular basis for the success of its hybridization with cucumber. Moreover, expanded gene families of C. hystrix were significantly enriched in "defense response," and C. hystrix harbored 104 nucleotide-binding site-encoding disease resistance gene analogs. Furthermore, 121 genes were positively selected, and 12 (9.9\%) of these were involved in responses to biotic stimuli, which might explain the high disease resistance of $C$. hystrix. The alignment of whole C. hystrix genome with cucumber genome and self-alignment revealed 45,417 chromosomespecific sequences evenly distributed on C. hystrix chromosomes. Finally, we developed four cucumber-C. hystrix alien addition lines and identified the exact introgressed chromosome using molecular and cytological methods. The assembled C. hystrix genome can serve as a valuable resource for studies on Cucumis evolution and interspecific introgression breeding of cucumber.
\end{abstract}

\section{Introduction}

Cucumis hystrix Chakr. $(2 \mathrm{n}=2 \mathrm{x}=24)$ is a wild perennial congener of cucumber (C. sativus $\mathrm{L}$., $2 \mathrm{n}=2 \mathrm{x}=14$ ) and melon (C. melo L., $2 \mathrm{n}=2 \mathrm{x}=24)$. It is a climber and grows in bushes on hills at $\sim 1 \mathrm{~km}$ above the mean sea level, particularly along the streams where the sunlight is

\footnotetext{
Correspondence: Sanwen Huang (huangsanwen@caas.cn) or Jinfeng Chen (jfchen@njau.edu.cn)

'State Key Laboratory of Crop Genetics and Germplasm Enhancement, Nanjing Agricultural University, 210095 Nanjing, China

${ }^{2}$ College of Horticulture, Qingdao Agricultural University, 266109 Qingdao, China

Full list of author information is available at the end of the article These authors contributed equally: Xiaodong Qin, Zhonghua Zhang, Qunfeng Lou
}

poor and humidity is high (Fig. 1a, left). It is geographically distributed in Southeast Asia, from South China to Myanmar, Thailand, Bangladesh, and Northeast India ${ }^{1}$. The fruit of $C$. hystrix has a cucumber-like and slightly sour taste (Fig. 1a, bottom right). The stem of an adult $C$. hystrix plant can gradually become semi-lignified and crack during development. Male and female flowers of C. hystrix (Fig. 1a, top and middle right, respectively) are almost identical to those of cucumber, but smaller. It can overwinter in the native regions.

C. hystrix has attracted much attention because of its cross-compatibility with cucumber ${ }^{2}$ as well as resistance to biotic stresses (e.g., root knot and downy mildew ${ }^{3}$ ) and tolerance of abiotic stresses (e.g., low sunlight ${ }^{4}$ and low

\section{(c) The Author(s) 2021}

(c) (i) Open Access This article is licensed under a Creative Commons Attribution 4.0 International License, which permits use, sharing, adaptation, distribution and reproduction cc) in any medium or format, as long as you give appropriate credit to the original author(s) and the source, provide a link to the Creative Commons license, and indicate if changes were made. The images or other third party material in this article are included in the article's Creative Commons license, unless indicated otherwise in a credit line to the material. If material is not included in the article's Creative Commons license and your intended use is not permitted by statutory regulation or exceeds the permitted use, you will need to obtain permission directly from the copyright holder. To view a copy of this license, visit http://creativecommons.org/licenses/by/4.0/. 
a

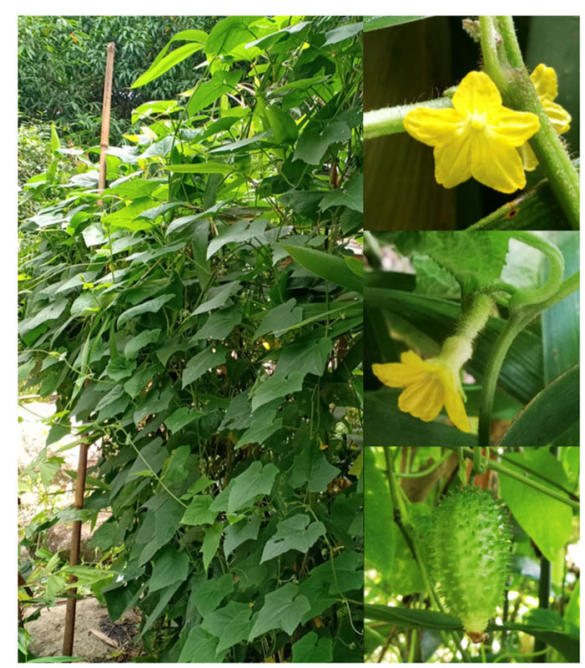

b

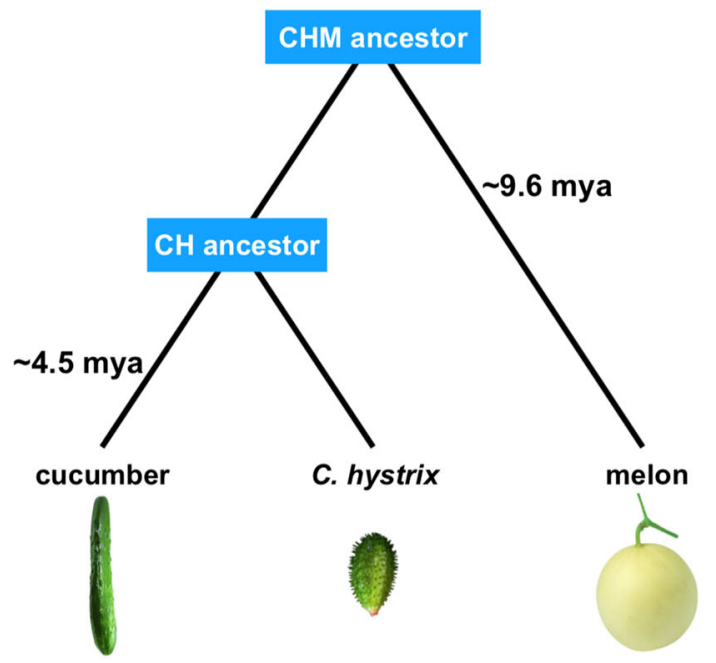

Fig. 1 Species information of Cucumis hystrix. a Morphological characteristics of C. hystrix. (left, adult plant; top right, male flower; middle right, female flower; bottom right, fruit). b Evolutionary relationships of cucumber (C), C. hystrix (H), and melon (M) and their divergence time (mya, million years ago)

temperature $\left.{ }^{5}\right)$. Cucumber is a valuable vegetable crop and widely consumed worldwide. However, the genetic base of cucumber has become increasingly narrow due to longterm and directed domestication, which is a hurdle in cucumber breeding ${ }^{6}$. Wild species possess abundant natural variations, which are absent in crops, and these variations can potentially enrich the gene pool of crops and further improve the desirable target traits ${ }^{7-10}$.

A new interspecific hybrid of Cucumis was successfully developed by doubling the chromosomes of the sterile F1 generation $(2 \mathrm{n}=2 \mathrm{x}=19)$ of cucumber and C. hystrix, giving rise to the allotetraploid Cucumis $\times$ hytivus J.-F. Chen \& J. H. Kirkbr. (C. hytivus, $2 \mathrm{n}=4 \mathrm{x}=38$ ). Successful hybridization of cucumber and $C$. hystrix proved to be a cornerstone of cucumber interspecific breeding. Following this, a number of introgression lines were developed through recurrent backcrossing of this artificial allotetraploid to cucumber, and some of these lines exhibited substantially increased disease resistance ${ }^{11}$. Genetic assessment of $C$. hytivus-derived inbred backcross lines indicated that the genetic diversity of cucumber was broadened $^{12}$.

Genome sequencing can identify abundant molecular markers with full coverage and high specificity and accuracy to trace the introgressed segments, which is crucial for interspecific introgression breeding. Therefore, high-quality genome assembly of C. hystrix is imperative to identify efficient interspecific hybrid materials and develop genetic resources for cucumber improvement.
Genomic data of flowering plants are rapidly accumulating $^{13}$. The cucumber whole genome-the first genome of a vegetable crop-was compiled in $2009^{14}$, which heralded the dawn of the genomics-directed era of vegetable breeding. The genome of melon, another economically important Cucumis crop, was also compiled 3 years later ${ }^{15}$. The evolutionary relationships of the three Cucumis species are shown in Fig. 1b. A three-way comparison can be used to track the potential events driving speciation. Previous studies assessed the phylogenetic relationships of Cucumis species using selected molecular markers ${ }^{16-19}$, cytological methods ${ }^{20,21}$, and genetic linkage maps ${ }^{22}$. Nevertheless, these methods have limited power to reveal the phylogenetic relationships among species, and considering the complex factors, such as incomplete lineage sorting, interspecific hybridizationinduced gene flow, and horizontal transfer, different data or computing methods may reveal diverse evolutionary history ${ }^{23-26}$. In this context, genome-scale comparative analysis can provide comprehensive and robust information for elucidating evolutionary events.

The genome of $C$. hystrix was preliminarily assembled in a previous study ${ }^{27}$, albeit with low coverage and continuity and without full annotation. This assembly is far from satisfactory, and the lack of a high-quality $C$. hystrix reference genome has indeed impeded the comparative genomic analyses of Cucumis species. To this end, the results of the present study provide an invaluable resource for uncovering the evolutionary events of Cucumis species and improving cucumber via interspecific hybridization. 


\section{Results}

\section{C. hystrix genome assembly and quality assessment}

The estimated genome size, heterozygosity, and repeat content of the $C$. hystrix genome were $416 \mathrm{Mb}, 0.78 \%$, and $53.5 \%$, respectively. We assembled the C. hystrix genome using a hybrid method with different datasets (Table S1). Supernova $^{28}$ was used to assemble the $10 \times$ genomic data of the recommended size using default parameters. Contig N50 (minimum contig length representing half of the total length of the assembly) of the Supernova assembly was $108 \mathrm{~kb}$, and its scaffold N50 (minimum scaffold length representing half of the total length of the assembly) was $7.6 \mathrm{Mb}$. We conducted further gap-filling, polishing, and scaffolding using self-corrected PacBio, pair-end, and mate-pair data. A general workflow of the assembly is presented in Fig. S1. We finally assembled $289 \mathrm{Mb}$ sequences-approximately $80 \mathrm{Mb}$ more than the previously published assembly ${ }^{27}$. The contig N50 was $221 \mathrm{~kb}$, and the scaffold N50 was $14 \mathrm{Mb}$, with a 100- and 277-fold improvement, respectively. Moreover, $90.4 \%$ of the assembled scaffolds were anchored and $88.2 \%$ were oriented on 12 pseudochromosomes based on 416 markers in a linkage map developed in a previous study ${ }^{27}$. The overall scaffold anchoring statistics are summarized in Table S2, and the final assembly statistics are summarized in Table 1 . The GC content was $33.12 \%$, and the repeat sequences constituted $48.7 \%$ of the genome, with long terminal repeats being the most abundant (19.64\%). Repeat statistics of the assembly are summarized in Table S3. We predicted 23,864 gene models using a hybrid method based on ab initio, homology alignment, and transcriptome sequencing of five tissues (root, stem, leaf, male flower, and ovary). The results of a simple comparison of genome assembly among the three Cucumis species (cucumber, C. hystrix, and melon) are summarized in Table S4. The genome size of C. hystrix was estimated

Table 1 Statistics of Cucumis hystrix draft assembly

\begin{tabular}{ll}
\hline Statistics & Value \\
\hline Total size of assembled contigs (bp) & $289,989,644$ \\
Number of contigs (>100 bp) & 6072 \\
Largest contig (bp) & $1,438,864$ \\
Contig N50 (bp) & 220,950 \\
Total size of assembled scaffolds (bp) & $297,500,035$ \\
Number of scaffolds (>300 bp) & 2284 \\
Largest scaffold (bp) & $20,059,872$ \\
Scaffold N50 (bp) & $14,064,021$ \\
Sequences anchored on chromosomes (bp) & $268,892,684(90.4 \%)$ \\
Sequences oriented on chromosomes (bp) & $262,424,878$ (88.2\%) \\
\hline
\end{tabular}

to be larger than that of cucumber but smaller than that of melon, and the total size of the assembled sequences was in the same order.

We evaluated the quality of the genome using various methods. There was acceptable consistency between the assembly and linkage groups (Fig. S2). We randomly selected a region of chromosome 2 and found that most of it was supported by considerable mate-pair reads (Fig. S3). Of the 1440 single-copy orthologous genes from BUSCO $^{29}$, respectively, 1307 (90.7\%) and 31 (2.2\%) were assigned as complete and fragmented in the C. hystrix draft genome. A total of 1323 (91.9\%) complete and 46 (3.2\%) fragmented single-copy orthologous genes were detected in 23,864 putative proteins. The BUSCO results were comparable to those of several other published genome assemblies of Cucurbitaceae species (Table S5). All assessment results indicated that the C. hystrix genome assembly was of high quality.

\section{Similarities among cucumber, C. hystrix, and melon at the nucleotide and protein levels}

We conducted comprehensive pairwise whole-genome alignments using the assembled genomes of cucumber, $C$. hystrix, and melon and annotated their proteomes. Specifically, $223.1 \mathrm{Mb}$ (74.9\%) sequences of C. hystrix were aligned to $199.0 \mathrm{Mb}(88.0 \%)$ sequences of cucumber, and $161.0(54.1 \%)$ and $160.2 \mathrm{Mb}(70.8 \%)$ sequences of $C$. hystrix and cucumber, respectively, showed one-to-one correspondence. Meanwhile, only 156.1 Mb (52.4\%) sequences of $C$. hystrix could be aligned to $172.3 \mathrm{Mb}$ (41.4\%) sequences of melon using the same alignment parameters, with only 111.5 (37.5\%) and $111.7 \mathrm{Mb}$ (26.8\%) sequences of $C$. hystrix and melon, respectively, showing one-to-one correspondence. Cucumber, $C$. hystrix, and melon genomes contained 25.3, 62.7, and $238.1 \mathrm{Mb}$ species-specific (no hits for either of the other two species) sequences, respectively. The average identity of the aligned sequences was $91.55 \%$ between C. hystrix and cucumber, $89.29 \%$ between $C$. hystrix and melon, and $89.56 \%$ between cucumber and melon.

We further examined the identity distribution of sequences showing one-to-one correspondence (Fig. 2a) and calculated the total and average length of the aligned sequences in each identity interval (Fig. 2c). C. hystrix shared a higher similarity median with cucumber than with melon. The median between $C$. hystrix and melon was low and that between cucumber and melon was comparable (Fig. 2a). C. hystrix and cucumber shared the most genomic sequences with high similarity (above $85 \%$ ). C. hystrix shared a longer average length of aligned sequences in each identity interval with cucumber than with melon (Fig. 2c). In addition, C. hystrix shared a higher average identity of protein reciprocal best hits (RBHs) with cucumber (96.56\%) than with melon 
a

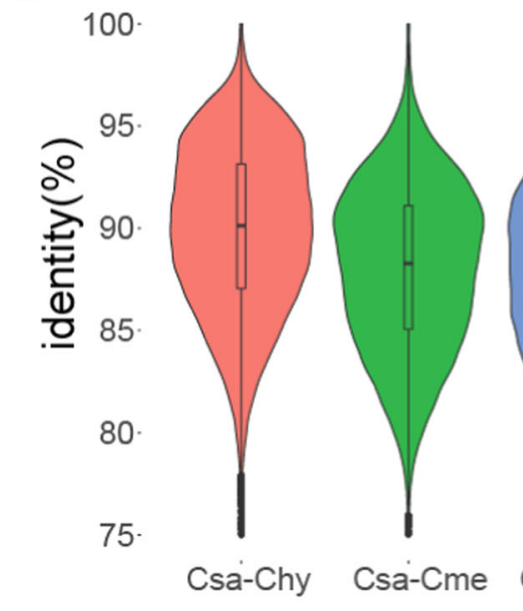

C

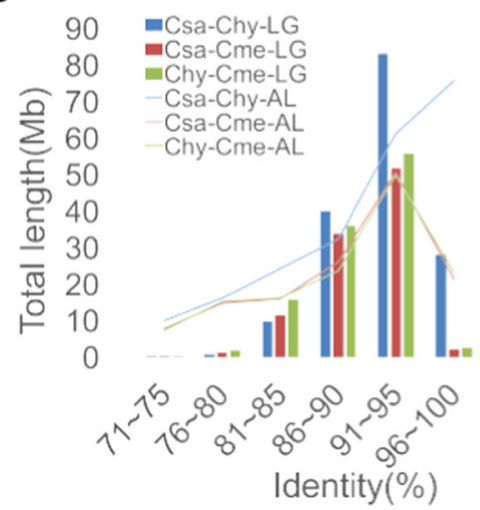

b
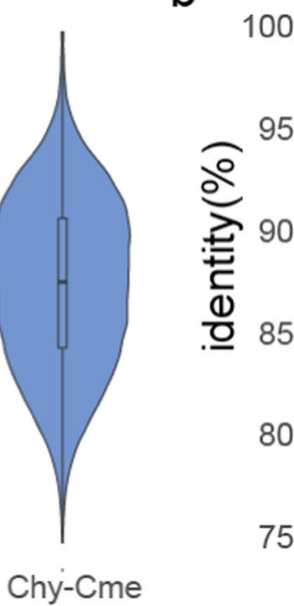

00.
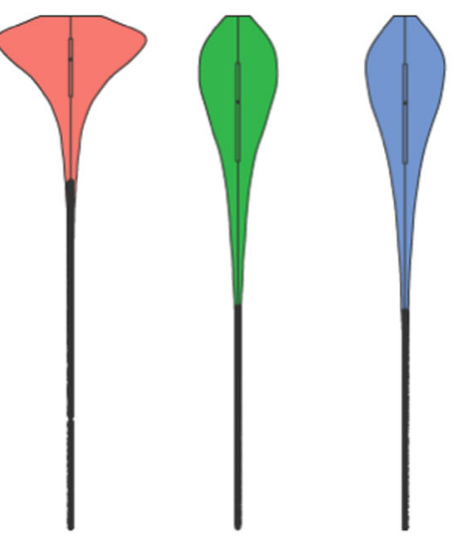

Csa-Chy

Csa-Cme

Chy-Cme

d

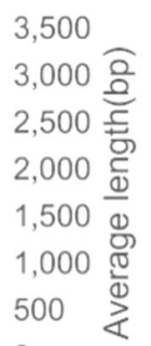

0

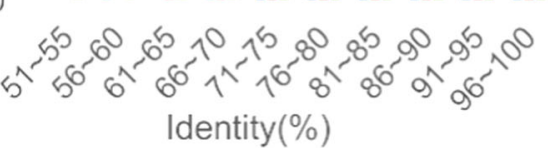

Fig. 2 Similarities among cucumber, Cucumis hystrix, and melon at the nucleotide and protein levels. a Identity distribution of the aligned segments with one-to-one correspondence. $\mathbf{b}$ Identity distribution of pairwise best-hit proteins. $\mathbf{c}$ Total and average length of each identity interval. d Total number of pairwise proteins in each identity interval. LG, length; AL, average length; Csa, Cucumis sativus; Chy, Cucumis hystrix; Cme, Cucumis melo

(94.34\%), and the average identity of RBHs between cucumber and melon was moderate (94.41\%). The similarity distribution of RBHs demonstrated that C. hystrix shared a significantly higher median with cucumber than with melon (Fig. 2b), and most proteins showed over 95\% similarity (Fig. 2d). The higher similarity of C. hystrix with cucumber at the DNA and protein level explained their close relationship and the cucumber-like phenotype of $C$. hystrix, providing a molecular basis for the successful hybridization between these two species.

\section{Genome collinearity of cucumber, $C$. hystrix, and melon}

We detected 16,916 RBHs between cucumber and $C$. hystrix, 16,131 RHBs between $C$. hystrix and melon, and 15,200 RHBs between cucumber and melon. We then used these RBHs to assess the collinearity among the three Cucumis species using McScanX ${ }^{30}$. Respectively, 119, 240, and 182 blocks with at least 5 RBHs were detected between $C$. hystrix and cucumber, $C$. hystrix and melon, and cucumber and melon. The average number of gene blocks between cucumber and C. hystrix was 137, almost two-fold the number between $C$. hystrix and melon (79) and more than two-fold the number between cucumber and melon (64). The largest block with the highest number of genes was also detected between cucumber and C. hystrix, which contained 960 orthologous gene pairs and covered $10.8 \mathrm{Mb}$ genomic sequences of C. hystrix on chromosome 6 and $9.4 \mathrm{Mb}$ genomic sequences of cucumber on chromosome 3. The statistics of RBHs and the detected blocks are summarized in Table S6. Detailed information of each block is presented in Tables S7-S9. Based on the position of the blocks detected, the overall collinearity across the whole genomes of the three Cucumis species is demonstrated in Fig. 3a. The primary syntenic relationship of the chromosomes was highly consistent with the previous reports ${ }^{27}$, detected by the comparison of linkage maps. C. hystrix showed the same karyotype as melon, but it shared fewer blocks and more average genes per block with cucumber microscopically, although their collinear blocks showed a 


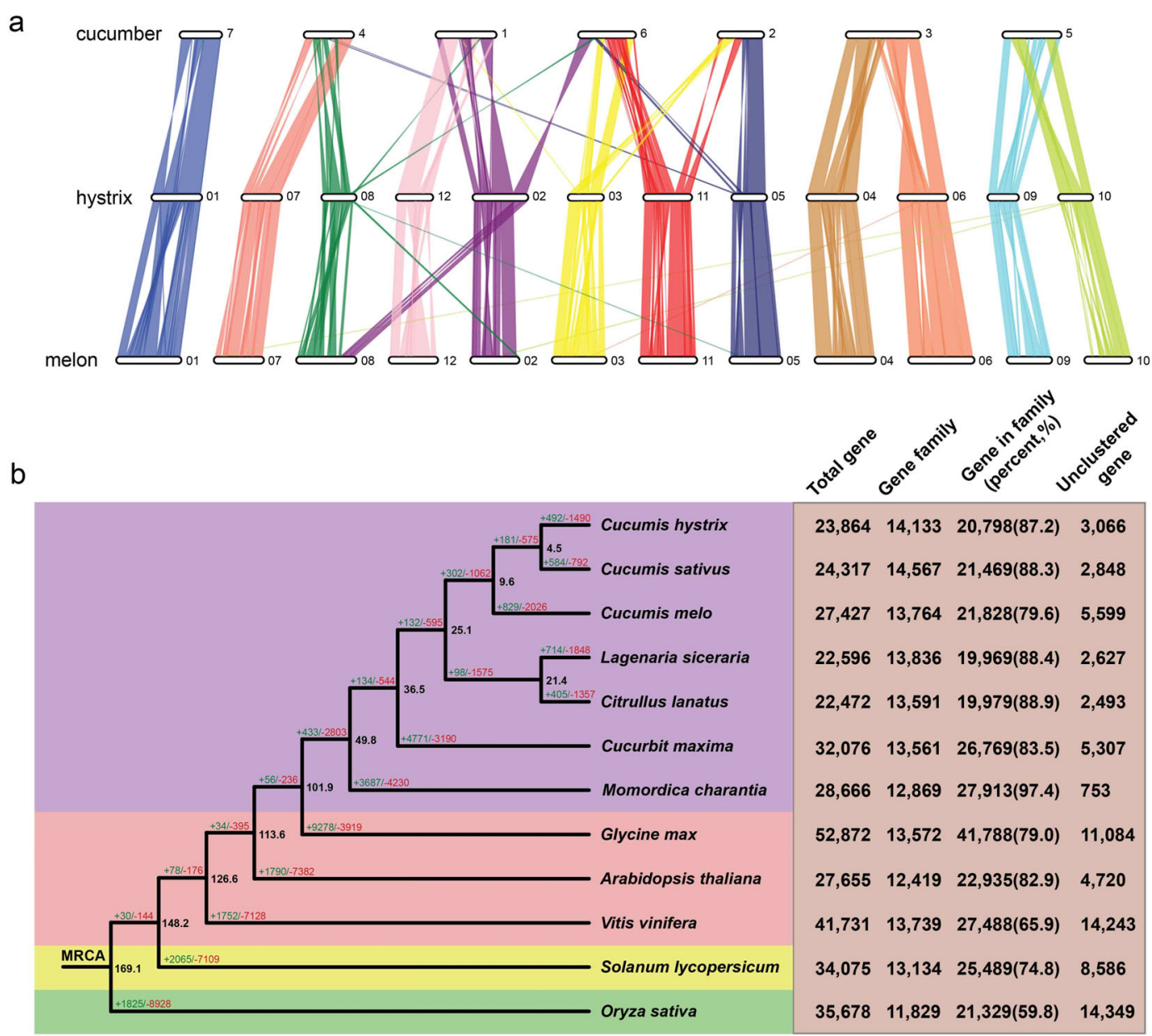

C

d
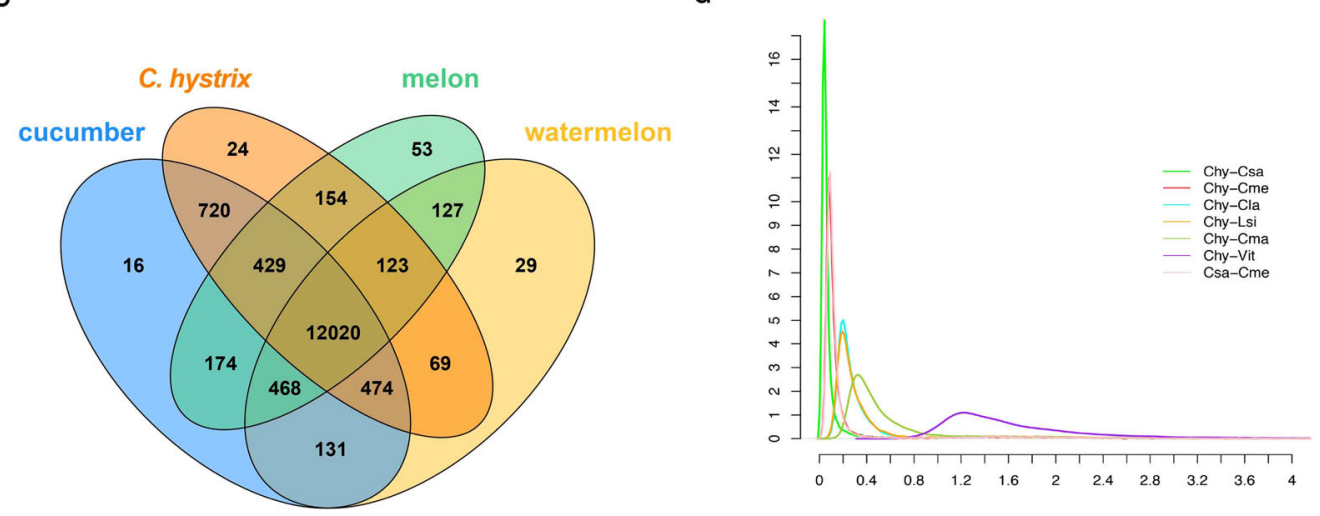

Fig. 3 Genome evolution of Cucumis hystrix. a Genome collinearity analysis of cucumber, Cucumis hystrix, and melon. Chromosome number is showed at the right end of each chromosome diagram. $\mathbf{b}$ Phylogenetic relationships of the 12 selected species and gene family evolution. The numbers of total genes, gene families, clustered genes, and unclustered genes are summarized in the right table. Black numbers at each node represent the estimated time of each divergent event. Green and red numbers along each branch indicate the number of expanded and contracted gene families, respectively. MRCA, most recent common ancestor. c Venn diagram of shared gene families among cucumber, $C$. hystrix, melon, and watermelon. d Ks distribution of synthetic orthologs of the selected species. Csa, C. sativus, C. hystrix, Cme, C. melo; Cla, Citrullus lanatus; Lsi, Lagenaria siceraria; Cma, Cucurbita maxima; Vit, Vitis vinifera 
complex, mosaic correspondence. These results indicate the occurrence of recent large-scale chromosomal rearrangements, which likely played a key role in cucumber speciation. Moreover, phylogenetic analyses based on the overall collinearity or robust karyotypes of species yield unreliable results.

\section{Phylogenetic tree and specific or expanded/contracted gene families in Cucumis species}

We clustered genes of the three Cucumis species, four non-Cucumis Cucurbitaceae species (bottle gourd, watermelon, squash, and bitter gourd), and five other species, including rosids (soybean, Arabidopsis, and grape), asterids (tomato), and monocots (rice), into 17,901 gene families using OrthoFinder ${ }^{31}$. The numbers of total genes, gene families, clustered genes, and unclustered genes are listed in the right orange table of Fig. 3b. We focused on the gene families of Cucumis species using watermelon as the outgroup. General statistics are presented as a Venn diagram (Fig. 3c). A total of 15,011 gene families with at least two genes were clustered, and the four selected Cucurbitaceae species shared 12,020 gene families. Cucumber, C. hystrix, and melon shared 12,449 gene families, which could be recognized as the core gene set of Cucumis species. A total of 429 clusters were specifically shared by Cucumis species. C. hystrix shared the most gene families with cucumber, reflecting their close relationship. Moreover, 24 clusters containing 64 genes were unique to $C$. hystrix.

We collated 304 single-copy genes of the 12 species into supergenes to construct a phylogenetic tree (Fig. 3b). C. hystrix was the closest relative of cucumber, and their common ancestor was placed in the same clade as melon, which is consistent with previous reports ${ }^{19}$. We then calculated the synonymous substitution rate of each collinear paralogous gene between and within several selected species. The density distribution indicated that $C$. hystrix shared the smallest peak with cucumber (Fig. 3d). We further estimated that $C$. hystrix and cucumber diverged from their common ancestor about 4.5 million years ago (mya), indicating that they had a relatively short divergence time.

Gene family expansion and contraction play significant roles in phenotypic adaption during speciation. Duplicated genes may enhance the metabolic pathways in which they participate and may also acquire novel functions-called neofunctionalization ${ }^{32-34}$. We conducted gene family expansion and contraction analysis of the shared gene families among the 12 selected species (Fig. 3b). There were 584/792, 492/1490, and 829/2026 expanded/contracted gene families in cucumber, $C$. hystrix, and melon, respectively. The top 20 Gene Ontology (GO) enrichment terms of the expanded gene families for each Cucumis species are shown in Fig. S4.
The most enriched and abundant function in C. hystrix was "defense response" (GO:0006952), which might protect this species from various abiotic or biotic stresses in the wild. "Organelle organization" (GO:0006996) was the most enriched function and "developmental process" (GO:0032502) was the most abundant function in cucumber. "DNA integration" was the most enriched function (GO:0015074) and "cellular metabolic process" (GO:0044237) was the most abundant function in melon. No overlap in function was noted among the expanded gene families of Cucumis species, indicating that their expansion may have driven Cucumis speciation.

\section{Positively selected genes (PSGs) in C. hystrix}

We identified 55, 121, and 92 PSGs in cucumber, $C$. hystrix, and melon, respectively (false discovery rate $<0.05$ ), using PosiGene ${ }^{35}$. Here, we focus on the PSGs in C. hystrix. We found that 93 (76.9\%) PSGs were singlecopy, which likely played important roles in C. hystrix speciation. We further conducted GO analysis of these PSGs and observed 18 enriched PSGs (Table 2). Two of these enriched processes were "response to biotic stimulus" (GO:0009607) and "defense response to other organisms" (GO:0098542), involving 12 genes, which likely enhanced the disease resistance of $C$. hystrix. For instance, the homolog of ChyUNG234630.1 in Arabidopsis thaliana (AT5G06720 and AtPRX53), which plays diverse roles in wound response, flower development, and syncytium formation, was found to be involved in response to nematode infection in soybean ${ }^{36}$ and $A$. thaliana $^{37}$. Moreover, the homolog of Chy3G060900.1 in A. thaliana (AT2G45180 and DRN1), a nonspecific lipid transfer protein, was found to be essential for resistance against various phytopathogens and tolerance to salt stress $^{38}$. The general information of these 12 genes is summarized in Table S10.

\section{Identification of resistance (R) gene analogs (RGAs) and evolutionary analysis of nucleotide-binding site (NBS)- encoding genes in Cucumis}

The $R$ genes play critical roles in the arms race of plant-pathogen interaction in the immune system of plants $^{39}$. We used RGAugury ${ }^{40}$ to identify the potential RGAs in the three Cucumis species. The total predicted RGA numbers for each species are listed in Table 3. Here, we focused on the R genes containing the NBS domain, which are the most frequently cloned and described genes in plants ${ }^{41,42}$. We detected 74,104 , and 84 RGAs in cucumber, $C$. hystrix, and melon, respectively. Genes with $<80 \%$ coverage of the NBS domain were excluded from the subsequent analysis, finally yielding 54,65 , and 51 genes. We anchored each NBS-encoding gene (excluding the genes on scaffolds) of $C$. hystrix to its pseudochromosomes (Fig. 4a). The results indicated that $39(60 \%)$ 
NBS-encoding genes were located on chromosomes 1, 5, and 9, with most exhibiting a clustered pattern, which is consistent with previous reports ${ }^{43-45}$. The remaining chromosomes were sporadically distributed on other chromosomes. There were no full-length NBS-encoding genes predicted on chromosomes 8 and 12 .

Table 2 Enriched Gene Ontology (GO) terms for positively selected genes in Cucumis hystrix

\begin{tabular}{|c|c|c|}
\hline GO ID & Description & $P$ value \\
\hline GO:0015691 & Cadmium ion transport & 0.000971 \\
\hline GO:0051351 & Positive regulation of ligase activity & 0.001243 \\
\hline GO:0051443 & $\begin{array}{l}\text { Positive regulation of ubiquitin-protein } \\
\text { transferase activity }\end{array}$ & 0.001243 \\
\hline GO:0032973 & Amino acid export & 0.001885 \\
\hline GO:0031398 & Positive regulation of protein ubiquitination & 0.002253 \\
\hline GO:1903322 & $\begin{array}{l}\text { Positive regulation of protein modification by } \\
\text { small protein conjugation or removal }\end{array}$ & 0.002653 \\
\hline GO:0009605 & Response to external stimulus & 0.004474 \\
\hline GO:1901658 & Glycosyl compound catabolic process & 0.004554 \\
\hline GO:0098542 & Defense response to other organisms & 0.004660 \\
\hline GO:0006218 & Uridine catabolic process & 0.005996 \\
\hline GO:0018160 & Peptidyl-pyrromethane cofactor linkage & 0.005996 \\
\hline GO:0090228 & $\begin{array}{l}\text { Positive regulation of red or far-red light } \\
\text { signaling pathway }\end{array}$ & 0.005996 \\
\hline GO:0008654 & Phospholipid biosynthetic process & 0.006707 \\
\hline GO:0046474 & Glycerophospholipid biosynthetic process & 0.006939 \\
\hline GO:0051340 & Regulation of ligase activity & 0.007586 \\
\hline GO:0051438 & $\begin{array}{l}\text { Regulation of ubiquitin-protein transferase } \\
\text { activity }\end{array}$ & 0.007586 \\
\hline GO:0009607 & Response to biotic stimulus & 0.008847 \\
\hline GO:0072527 & $\begin{array}{l}\text { Pyrimidine-containing compound metabolic } \\
\text { process }\end{array}$ & 0.008851 \\
\hline
\end{tabular}

To study the evolution of the predicted genes containing the full-length NBS domain in the Cucumis species, we constructed a phylogenetic tree using the sequences of the conserved NB-ARC (PF00931) domain (Fig. 4b). The sequences formed four main clusters, namely RPW8, CNL I, CNL II, and TNL. RPW8 was the smallest cluster, with three genes in each Cucumis species. The CNL I cluster was significantly expanded in C. hystrix (11), containing almost two-fold more genes than in cucumber (6) and melon (6). The number of genes in the CNL II cluster was comparable between cucumber (22) and C. hystrix (25), but the number in melon (13) was half the number in the other two species. The number of genes in the TNL cluster was comparable among the three Cucumis species, being 23 in cucumber, 26 in C. hystrix, and 29 in melon. Moreover, a subclade of TNL was expanded in C. hystrix (Fig. 4b). In addition, the TNL cluster was located between two clusters on chromosomes 5 and 9, and the CNL II cluster between two clusters on chromosomes 1 and 4 . The expanded NBS-encoding genes in C. hystrix might explain its high disease resistance to some extent.

\section{Development and identification of cucumber-C. hystrix alien additional lines (CH-AALs)}

AALs are powerful tools for genome structure research and functional genomics and may serve as a bridge to introgress useful genes into recurrent parents in crop breeding. We developed four $\mathrm{CH}$-AALs with different $C$. hystrix chromosomes by recurrently backcrossing the artificial allotetraploid to cucumber. The detailed process is illustrated in Fig. S5. These lines were morphologically distinct, and the typical phenotype of each $\mathrm{CH}$-AAL is shown in Fig. S6.

To verify the exact identity of each alien chromosome in each $\mathrm{CH}$-AAL, we first developed chromosome-specific markers for $C$. hystrix and performed polymerase chain reaction (PCR) for each line. A total of 45,417 chromosome-specific sequences of $C$. hystrix were identified through inter- and intraspecific whole-genome alignment, ranging from 28 to $59,678 \mathrm{bp}$. Of these,

Table 3 Number of resistance genes in the three Cucumis species

\begin{tabular}{|c|c|c|c|c|c|c|c|c|c|c|c|}
\hline \multirow[t]{2}{*}{ Species } & \multicolumn{8}{|c|}{ NBS encoding } & \multirow[t]{2}{*}{ RLP } & \multirow[t]{2}{*}{ RLK } & \multirow[t]{2}{*}{ TM-CC } \\
\hline & NBS & CNL & TNL & $\mathrm{CN}$ & TN & NL & TX & Other & & & \\
\hline C. sativus & 5 & 16 & 15 & 3 & 3 & 21 & 5 & 6 & 49 & 436 & 122 \\
\hline C. hystrix & 20 & 23 & 16 & 8 & 2 & 18 & 7 & 10 & 55 & 347 & 130 \\
\hline C. melo & 11 & 13 & 17 & 5 & 5 & 19 & 9 & 5 & 40 & 359 & 103 \\
\hline
\end{tabular}

$C C$, coiled-coil; $L R R$, leucine-rich repeat; $L y S M$, lysin motif; $N B-A R C$, nucleotide binding-activity regulated cytoskeleton; $N B S$, nucleotide-binding site; $R G A$, resistance gene analog; $R L K$, receptor-like kinase; $R L P$, receptor-like protein; $S T T K$, serine/threonine and tyrosine kinase; $T I R$, Toll/interleukin-1 receptor; $T M$, transmembrane; $C N L$, CC-NBS-LRR; TNL, TIR-NBS-LRR; CN, CC-NBS; TN, TIR-NBS; NL, NBS-LRR; TX, TIR-unknown domain; Other, CC or TIR; RLP, TM-LRR, TM-LysM; RLK, TM-LRR-STTK, and TMLySM-STTK 

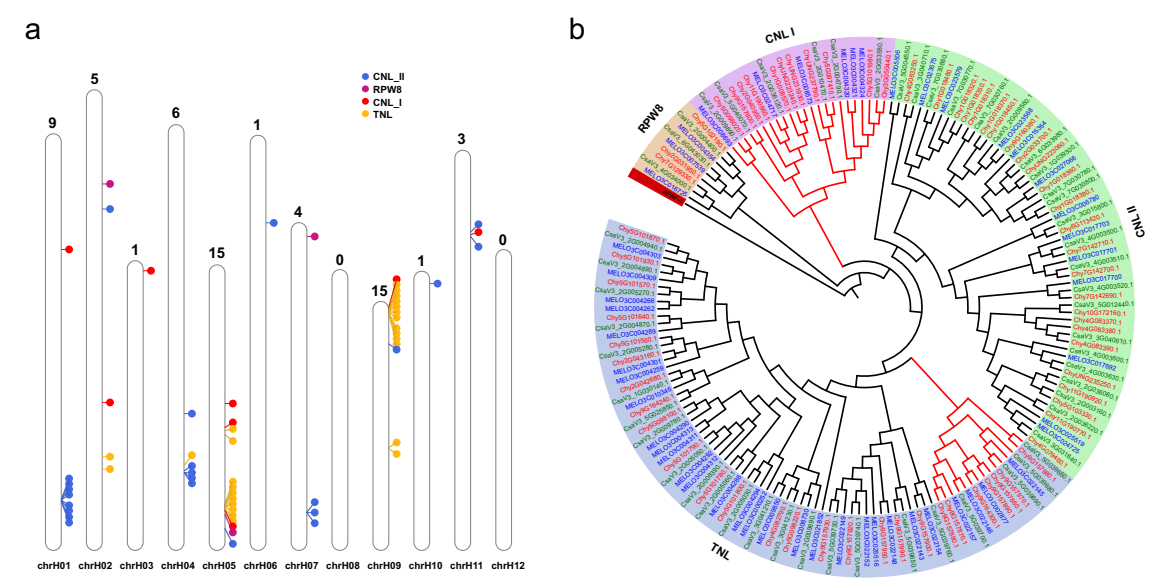

Fig. 4 Nucleotide-binding site (NBS)-encoding gene families of cucumber, Cucumis hystrix, and melon. a NBS gene distribution on each C. hystrix chromosome. The total number of NBS genes on each chromosome is labeled above. Dots with different colors represent different types of NBS genes. b Phylogenetic tree of NBS genes in cucumber, C. hystrix, and melon. Expanded clade/subclade in C. hystrix is labeled in red. Gene IDs of cucumber, C. hystrix, and melon are labeled in green, red, and blue, respectively. APAF-1 was used as the outgroup

9218 sequences were over $400 \mathrm{bp}$ and evenly distributed on each chromosome (Fig. S7a). Chromosome-specific sequences of cucumber were also identified and found to be evenly distributed on each chromosome (Fig. S7b). We selected 36 C. hystrix chromosome-specific sequences as markers (three on each chromosome) to design primers (Table S11). We conducted PCR for C. hystrix and cucumber, and all selected markers produced a chromosome-specific band in C. hystrix (Fig. S8). We selected one marker from each chromosome to conduct PCR for all CH-AALs (Fig. 5a). CH-AAL01 specifically produced bands for chrH06 and chrH09 (Fig. 5a, first from top). CH-AAL02 specifically produced bands for chrH08 and chrH10 (Fig. 5a, second from top). CHAAL03 produced a single band from chrH06 (Fig. 5a, third from the top). CH-AAL04 produced bands for chrH06 and chrH10 (Fig. 5a, fourth from top). The chromosome-specific bands produced by each $\mathrm{CH}-\mathrm{AAL}$ reflected introgression of $C$. hystrix segments into cucumber.

We further confirmed the identity of the alien chromosomes using next-generation sequencing (NGS) and fluorescence in situ hybridization (FISH). NGS reads (150 bp read length) of each $\mathrm{CH}-\mathrm{AAL}$ were aligned to the $C$. hystrix genome, and the number of highly similar reads ( $>99 \%$ identity with an alignment length of at least $145 \mathrm{bp}$ ) in each sliding window was determined (Fig. 5b). Chromosomes chrH06 and chrH09 were covered by a large number of highly similar reads showing a continuous pattern in CH-AAL01 (Fig. 5b, first from top). FISH signals of chrH06 and chrH09 were also detected in this line (Fig. 5c, first from left; Fig. S9a, b). The NGS and FISH results were consistent with the PCR results. Therefore, we confirmed that $\mathrm{CH}$-AAL01 received chrH06 and
chrH09 from C. hystrix. The identity of the introgressed C. hystrix chromosomes in the remaining three $\mathrm{CH}$-AALs was also verified using the same method (Fig. 5b, c and Fig. S9), and the detailed process is described in Materials and methods. All NGS and FISH results were consistent with the corresponding PCR results. Collectively, we successfully verified the exact identity of each $C$. hystrix chromosome in all $\mathrm{CH}$-AALs using different methods. The developed chromosome-specific markers may be used to efficiently screen for additional interspecific materials between $C$. hystrix and cucumber, serving as a bridge to enrich the cucumber gene pool.

\section{Discussion}

Phylogenetic relationships are key factors in determining the success of interspecific hybridization and the efficiency of genetic material exchange (introgression $)^{46,47}$. C. hystrix has a $2 n=2 \mathrm{x}=24$ karyotype-the same as melon-and they generally show a good genome collinearity. Meanwhile, cucumber has a distinct $2 \mathrm{n}=2 \mathrm{x}$ $=14$ karyotype. However, we found that $C$. hystrix shares better synteny with cucumber. The overall chromosome correspondence among the three Cucumis species tested in this study corroborated the previous reports ${ }^{27}$. Furthermore, we confirmed that $C$. hystrix is phylogenetically closer to cucumber than to melon at the molecular level based on the results of comprehensive genome-scale analysis, which explains the cucumber-like phenotype of C. hystrix. These findings further indicate that phylogenetic relationships based on karyotypes or overall collinearity can be misleading, and it is better to construct a robust phylogenetic tree at the molecular level to clarify the relationships among species, which is of high value for evolutionary studies and interspecific breeding. In 
a
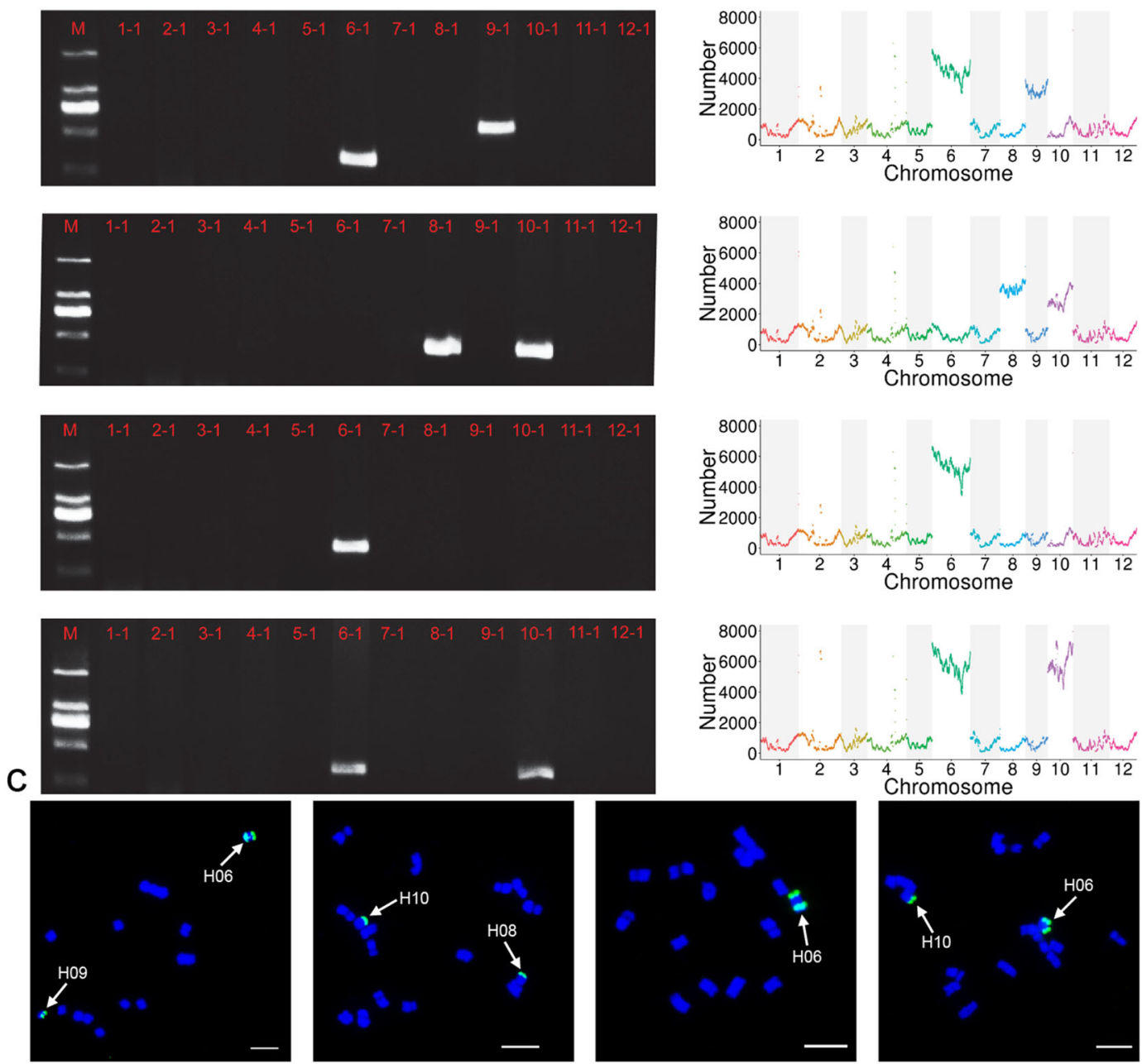

Fig. 5 Verification of the exact identity of each alien chromosome in cucumber-C. hystrix alien additional lines (CH-AALs). a Specific polymerase chain reaction band(s) of selected primer pair(s) for each CH-AAL (CH-AAL01-CHAAL04, from top to bottom). b Number of highly similar reads aligned to the $\mathrm{C}$. hystrix genome in sliding windows (1 Mb in size) for each $\mathrm{CH}-\mathrm{AAL}$ (CH-AAL01-CH-AAL04, from top to bottom). $\mathbf{c}$ Fluorescence in situ hybridization signal of introgressed C. hystrix chromosome(s) in each CH-AAL (CH-AAL01-CH-AAL04, from left to right)

addition, large-scale chromosome rearrangements, such as Robertsonian translocation, can drive speciation ${ }^{48}$. The complex events that shaped the evolution of seven pairs of chromosomes in cucumber from the 12 ancestral ones likely occurred gradually. However, this gives rise to other questions-were there any other phylogenetically intermediate species between $C$. hystrix and cucumber, and if so, do they still exist? It would be interesting and important to explore the answers, which would benefit the evolutionary studies and introgression breeding of Cucumis species.

Crops originate from their wild ancestors through domestication, during which artificial selection acts as a powerful driver shaping the crop genomes as well as their morphological characteristics and growth habits beneficial to humans ${ }^{49}$. The genetic base of cucumber, an economically important vegetable crop, has become extraordinarily narrow due to long-term domestication and recurrent use of limited variation during breeding ${ }^{6}$. As opposed to melon, which has been independently domesticated multiple times and has numerous crossfertile wild ancestors with a wide distribution from Asia to Africa $^{50}$, cucumber has a single cross-fertile wild ancestor originating from India, named C. sativus var. hardwickii, and the domestication of cucumber is limited to India ${ }^{19}$. Thus, cucumber breeding based only on intraspecific variation has encountered a bottleneck. In this light, successful interspecific hybridization of cucumber with its 
close wild relative $C$. hystrix provides an excellent opportunity to introgress novel genes, specifically those related to biotic or abiotic stress responses, in cucumber. In this study, we conducted comparative genomic analysis of cucumber, $C$. hystrix, and melon and demonstrated that gene families involved in defense response (e.g., NBSLRR) have significantly expanded in C. hystrix compared to those in cucumber and melon. A considerable number of PSGs in C. hystrix responded to biotic stimuli compared to those in the other selected Cucurbitaceae species. Finally, we developed and verified four phenotypically distinct cucumber lines introgressed for different $C$. hystrix chromosomes, which may serve as a bridge for introgressing novel genes from $C$. hystrix to cucumber.

Crop breeding has entered a new era in which genomic information has become increasingly pivotal ${ }^{51,52}$. In this study, we developed numerous chromosome-specific markers through the assembly of $C$. hystrix draft genome. We verified the specificity of these markers and found that they were evenly distributed on each $C$. hystrix chromosome, which could be of great significance for efficiently and unambiguously tracing the segments introgressed from $C$. hystrix to cucumber. Collectively, our findings provide valuable resources and data for evolutionary studies on Cucumis and lay a foundation for efficient cucumber breeding via interspecific hybridization.

\section{Materials and methods}

Plant material, DNA and RNA extraction, and sequencing

Seeds of $C$. hystrix were collected by Professor Jinfeng Chen from Xishuangbanna (Yunnan, China) and selfpollinated for several generations by germinating on Petri dishes at $25^{\circ} \mathrm{C}$. High-quality DNA was extracted from fresh young leaves using a modified cetyltrimethylammonium bromide method. A 10× Genomics Chromium library was constructed according to the manufacturer's instructions within droplets containing Gel Beads-in-Emulsion (GEMs) mixed with DNA and polymerase for wholegenome amplification. DNA was sheared within each GEM, and each molecule was tagged with an identical barcode (linked reads). As a result, $35 \mathrm{~Gb}$ reads with a length of $150 \mathrm{bp}$ were generated by sequencing the library on the Illumina HiSeq X Ten platform. One pair-end with an insert size of $500 \mathrm{bp}$ and four mate-pair "jumping libraries" with insert sizes of 2 and $8 \mathrm{k}$ were constructed following the standard Illumina protocol. The reads were sequenced on the Illumina Hiseq 2500 platform, and $27 \mathrm{~Gb}$ of pair-end (read length, $250 \mathrm{bp}$ ) and $49 \mathrm{~Gb}$ of mate-pair (read length, 125-150 bp) sequences were generated. For PacBio data sequencing, the genomic DNA was sheared into segments of $15-40 \mathrm{~kb}$, and a singlemolecule real-time library was constructed following the PacBio-recommended method. We obtained $10 \mathrm{~Gb}$ of
PacBio sequences with an average length of $5.6 \mathrm{~kb}$. The corresponding statistics are summarized in Table S1.

RNA from five C. hystrix tissues (root, stem, leaf, ovary, and male flower) was extracted using the QIAGEN RNeasy Plant Mini Kit, following the manufacturer's instructions (QIAGEN, Valencia, CA, USA). Strandspecific RNA-sequencing (RNA-Seq) libraries were constructed using the protocol described by Zhong et al. ${ }^{53}$. The RNA-Seq libraries were sequenced on the Illumina HiSeq X system with a pair-end read length of $150 \mathrm{bp}$. We obtained 8.5, 9.3, 9.8, 10.6, and 9.6 Gb sequences from the five tissues, respectively.

The detailed process of CH-AAL development is presented in Fig. S5. The protocol for DNA sample preparation was the same as above. The libraries were constructed according to the manufacturer's instructions. Resequencing of these libraries generated $8.5(\mathrm{CH}-$ AAL01), 9.2 (CH-AAL02), 10.6 (CH-AAL03), and $12.3 \mathrm{~Gb}$ (CH-AAL04) pair-end reads with a length of $150 \mathrm{bp}$ on the Novaseq 6000 sequencing system.

\section{Genome assembly and quality assessment}

The genome size, heterozygosity, and repeat content of the $C$. hystrix genome were estimated using $\mathrm{GCE}^{54}$. First, $10 \times$ genomic-linked reads were assembled using Supernova ${ }^{28}$. The read number used for assembly was calculated according to the recommended depth. We fed Pilon $^{55}$ with the PE250 pair-end data, which were filtered by fastp ${ }^{56}$ according to the base quality, length, and overlapping information, to polish the scaffolds generated by Supernova. To fill the gaps in the polished scaffolds, we first assembled super-reads by running $\mathrm{MaSuRCA}^{57}$ on all raw $\mathrm{PE}$ reads. PacBio long reads were then self-corrected using $\mathrm{Canu}^{58}$. Super-reads and the corrected long reads were merged and fed to PBjelly ${ }^{59}$ for gap-filling. The Pilon polishing step was repeated on the gap-filled scaffolds. We ran SSPACE ${ }^{60}$ on the 2 and $8 \mathrm{k}$ mate-pair libraries, which were filtered by NextClip ${ }^{61}$ to further merge the secondary polished scaffolds. We conducted e-PCR ${ }^{62}$ on the markers from the linkage group developed by Yang et al. ${ }^{27}$ to locate them on the scaffolds. Finally, based on the marker location information on the scaffolds and linkage groups, the scaffolds were anchored, ordered, and oriented along 12 pseudochromosomes using ALLMAPS ${ }^{63}$. The assembly workflow is summarized in Fig. S1.

We used three methods to evaluate the quality of our genome assembly. We first checked the consistency of the assembly with a linkage map using ALLMAPS. The matepair reads of the 2 and $8 \mathrm{k}$ library were aligned to the assembly using the Burrows-Wheeler Aligner ${ }^{64}$, and a $2.5 \mathrm{Mb}$ segment of chromosome 2 was selected as an example. We further examined the coding region completeness of the genome assembly and the other selected Cucurbitaceae species with $\mathrm{BUSCO}^{29}$. 


\section{Genome annotation}

We first detected the repeat sequences in the final assembly using RepeatModeler. De novo-detected repeats were then combined with the TIGR plant repeats database (http://plantrepeats.plantbiology.msu.edu) and repeated with RepeatMasker (http://repeatmasker.org).

A hybrid method of transcriptome mapping, ab initio, and homologous alignment was used for gene prediction of the repeat-masked assembly. Transcriptomic data from five tissues were mapped to the reference with HISAT2 ${ }^{65}$ and assembled using stringtie ${ }^{66}$. The output transcripts were then fed to PASA (http://pasa.sourceforge.net) for further processing. Three tools, including GlimmerHMM $^{64}$, Augustus ${ }^{66}$, and SNAP $^{67}$, were used for ab initio prediction. Non-redundant plant proteins from Uniprot (http://www.uniprot.org) were downloaded and aligned to the assembly with Wise ${ }^{67-70}$. Finally, EVidenceModeler $^{71}$ was used to integrate the evidence detected and generated gene structures based on their weights. The completeness of the final predicted gene set was evaluated using BUSCO $^{29}$.

\section{Comparative genomics}

The whole genomes Cucumis species were aligned using Mummer $4.0^{72}$ with default parameters. RBHs were identified using a script that depends on BLAST $+{ }^{73}$ and then fed to McScanX ${ }^{30}$ to detect syntenic blocks between each pair of species.

To calculate the synonymous substitution rate (Ks) of the homologous gene pairs in the selected species, we first conducted all-vs-all BLASTP $(E$ value $<1 \mathrm{e}-5)$. Collinear homologous gene pairs within or between species were identified using McScanX ${ }^{30}$. We then aligned their coding sequences (CDSs) using ParaAT ${ }^{74}$. Finally, the Ks value of each homologous gene pair was calculated using KaKs_Calculator ${ }^{75}$.

Orthofinder ${ }^{31}$ was used to identify gene families of $C$. hystrix, cucumber, and melon, as well as the selected 9 species, including four non-Cucumis Cucurbitaceae (bottle gourd, watermelon, squash, and bitter gourd), four other dicot species, including rosids (soybean, Arabidopsis, and grape) and asterids (tomato), and one monocot species (rice). Gene family expansion/contraction was detected with $\mathrm{Café}^{76}$ using a probabilistic graphical model. Next, 304 single-copy genes identified by OrthoFinder in the 12 aforementioned species were fed into $\mathrm{RAxML}^{77}$ to clarify their phylogenetic relationships. To estimate the divergence time of the species, we used the MCMCtree program of PAML ${ }^{78}$. GO enrichment analysis was performed on the OmicShare online platform (http:// www.omicshare.com/tools).

PSGs of C. hystrix were identified by feeding the CDSs of nine Cucurbitaceae species, including cucumber, $C$. hystrix, melon, watermelon, bottle gourd, Cucurbita maxima, monk fruit, bitter gourd, and wax gourd, to PosiGene ${ }^{35}$. We used cucumber as the anchor species. RGAs were predicted by RGAugury ${ }^{40}$. NBS-encoding genes were then extracted for further analysis. Genes with over $80 \%$ coverage of the NB-ARC (PF00931) domain were aligned using MUSCLE ${ }^{79}$. To illustrate the evolutionary history of the full-length NBS-encoding genes of the three Cucumis species, we constructed a phylogenetic tree using IQ-TREE ${ }^{80}$. The resulting Newick tree was fed to $\mathrm{iTOL}^{81}$ for visualization and further editing.

\section{Genome data collection}

The genome data of cucumber, melon, watermelon, bottle gourd, C. maxima, and wax gourd were downloaded from the Cucurbit Genomics Database (http:// cucurbitgenomics.org). The data of Luffa cylindrical ${ }^{82}$ and Momordica charantia ${ }^{83}$ were downloaded according to the corresponding reference. The cucumber genome version 3 and the melon genome version 3.5.1 were used in comparative genomics. Other genomic data were downloaded from the NCBI database.

\section{Identification of CH-AALs}

For the amplification of $C$. hystrix-specific molecular markers, we first extracted the unmatched sequences of $C$. hystrix from its alignment with the cucumber genome. These species-specific sequences were then realigned to the $C$. hystrix genome using BLASTN with default parameters. Sequences showing no hits with other chromosomes were recognized as chromosome-specific markers. We selected three markers evenly distributed on each chromosome to verify their specificity using PCR (Fig. S8). Twelve markers, one from each chromosome, were used for PCR of the CH-AALs.

\section{Analysis of the NGS data of CH-AALs}

We first selected $\sim 2 \times$ reads from the generated NGS data of each $\mathrm{CH}-\mathrm{AAL}$ and aligned these to the $C$. hystrix draft genome using BLASTN ( $E$ value $<1 \mathrm{e}-5$ ). The best hit of each read was extracted from the BLASTN results. Reads with an alignment length $>145 \mathrm{bp}$ and sequence similarity above $99 \%$ were considered to be from $C$. hystrix. Finally, the number of reads from $C$. hystrix in each $1 \mathrm{Mb}$ window with a step size of $10 \mathrm{~kb}$ was counted and visualized with an in-house $\mathrm{R}$ script.

\section{FISH}

We used the whole-genome DNA of C. hystrix as probes to conduct FISH in each CH-AAL and found one or two signals in all lines (Fig. 5c). To further verify the identity of the alien chromosomes, we designed different schemes. There were two alien chromosome signals in CH-AAL01 (Fig. 5c, first left). We used the oligo-probe pool of chromosome 5 (oligo C5) from cucumber ${ }^{84}$ to 
conduct FISH and found that one of the alien chromosomes showed a signal (Fig. S9a). Chromosome 5 of cucumber corresponded to chromosomes 9 and 10 of $C$. hystrix (Fig. 3a). According to our previous FISH results, only chromosomes 8,10 , and 12 showed $45 \mathrm{~S}$ signals in $C$. hystrix $^{85}$. Because this chromosome showed no $45 \mathrm{~S}$ signal (Fig. S9a), we concluded that it was chromosome 9 from C. hystrix. Collinearity analysis in this study (Fig. 3a) demonstrated that a 6-6.5 Mb region of chromosome 3 of cucumber corresponded to a segment of chromosome 6 of C. hystrix (to clearly show collinearity, we reversed chromosome 3 of cucumber in Fig. 3a). We designed oligo probes for this region (oligo C3-a) from cucumber to conduct FISH in CH-AAL01. Another alien chromosome showed a hybridization signal (Fig. S9b), which was determined to be chromosome 6 of C. hystrix. CH-AAL02 showed two alien chromosome signals (Fig. 5c, second from left). We used the oligo-probe pool of chromosome 4 from cucumber (oligo C4) ${ }^{86}$ to conduct FISH and found that one of them showed a signal (Fig. S9c). Chromosome 4 of cucumber corresponded to chromosomes 5,7 , and 8 of C. hystrix (Fig. 3a). Because this alien chromosome showed a 45S signal (Fig. S9c), we concluded that it was chromosome 8 from C. hystrix. The oligo $\mathrm{C} 5$ of $\mathrm{CH}$ AAL02 showed a signal in another alien chromosome (Fig. 9d) but no 45S signal (Fig. S9c). Therefore, it was determined to be chromosome 10 from $C$. hystrix. The oligo $\mathrm{C} 3$-a of $\mathrm{CH}$-AAL03 showed one alien chromosome signal (Fig. 5c, second from right), which was determined to be chromosome 6 from C. hystrix (Fig. S9e). $\mathrm{CH}-$ AAL04 showed two alien chromosome signals (Fig. 5c, right), and one of them was a C3-a signal (Fig. S9f). The oligo $\mathrm{C} 5$ of $\mathrm{CH}-\mathrm{AAL} 04$ showed a signal in another alien chromosome and a $45 \mathrm{~S}$ signal (Fig. S9g), which were determined to be chromosomes 6 and 10 from C. hystrix, respectively. The protocols for probe synthesis and FISH have been described by Zhao et al. ${ }^{84}$ and Bi et al. ${ }^{86}$.

\section{Acknowledgements}

This work was partially supported by the National Key Research and Development Program of China (\#2018YFD1000804), the National Natural Science Foundation of China (Key Program, \#31430075), the Belt and Road innovation cooperation project (\#BZ2019012), the National Key Research and Development Program of China (\#2016YFD0100204-25), the Jiangsu Agricultural Innovation of New Cultivars (\#PZCZ201719), and by a project funded by the Priority Academic Program Development of Jiangsu Higher Education Institutions.

\footnotetext{
Author details

'State Key Laboratory of Crop Genetics and Germplasm Enhancement, Nanjing Agricultural University, 210095 Nanjing, China. ${ }^{2}$ College of Horticulture, Qingdao Agricultural University, 266109 Qingdao, China. ${ }^{3}$ Institute of Vegetables and Flowers, Chinese Academy of Agricultural Sciences, 100081 Beijing, China. ${ }^{4}$ College of Horticulture and Landscape, Henan Institute of Science and Technology, 453003 Xinxiang, China. ${ }^{5}$ Agricultural Genomics Institute, Chinese Academy of Agricultural Sciences, 518120 Shenzhen, China
}

\section{Author contributions}

J.F.C. and H.S.W. designed the study and led the research. Z.H.Z. and X.D.Q. conducted the bioinformatic analysis. X.D.Q. wrote the paper. Y.C.X. and Q.L. assisted in comparative genomics and genome annotation, respectively. X.D. Q., S.Q.Y., L.X., and J.G.Z. grew plants and performed sampling for sequencing with the help of J.L., L.X., and M.X.L. X.K.Z. contributed to the creation and identification of alien additional lines under the direction of Q.F.L. and X.D.Q.

\section{Data availability}

Raw sequencing reads used are deposited in the Sequence Read Archive database under the accession number PRJNA649392. The final genome assembly and annotation information can be downloaded at https://doi.org/ 10.6084/m9.figshare.13377671.

\section{Conflict of interest}

The authors declare no competing interests.

Supplementary information The online version contains supplementary material available at https://doi.org/10.1038/s41438-021-00475-5.

Received: 29 September 2020 Revised: 30 December 2020 Accepted: 7 January 2021

Published online: 01 March 2021

\section{References}

1. Joseph John, K. et al. On the taxonomic status, occurrence and distribution of Cucumis hystrix Chakrav. and Cucumis muriculatus Chakrav. (Cucurbitaceae) in India. Genet. Resour. Crop Evol. 65, 1687-1698 (2018).

2. Chen, J. et al. Successful interspecific hybridization between Cucumis sativus $L$. and C. hystrix Chakr. Euphytica 96, 413-419 (1997).

3. Chen, J. et al. Some disease resistance tests in Cucumis hystrix and its progenies from interspecific hybridization with cucumber. Progress in Cucurbit Genetics and Breeding Research Proceedings of Cucurbitaceae 2004, the 8th EUCARPIA Meeting on Cucurbit Genetics and Breeding, 189-196 (Olomouc, 2004).

4. Qian, C. et al. Several photosynthetic characters of the synthetic species Cucumis hytivus Chen \& Kirkbride under weak light condition. Plant Physiol. Commun. 38, 336-338 (2002).

5. Zhuang, F. et al. Responses of seedlings of Cucumis hytivus and progenies to low temperature. JNAU 25, 27-30 (2002).

6. Qi, J. et al. A genomic variation map provides insights into the genetic basis of cucumber domestication and diversity. Nat. Genet. 45, 1510-1515 (2013).

7. S. D, T. \& S. R., M. Seed banks and molecular maps: unlocking genetic potential from the wild. Science 277, 1063-1066 (1997).

8. Zamir, D. Improving plant breeding with exotic genetic libraries. Nat. Rev. Genet. 2, 983-989 (2001).

9. Govindaraj, M. et al. Importance of genetic diversity assessment in crop plants and its recent advances: an overview of its analytical perspectives. Genet. Res. Int. 2015, 1-14 (2015).

10. Dempewolf, H. et al. Past and future use of wild relatives in crop breeding. Crop Sci. 57, 1070-1082 (2017)

11. Zhou, X. et al. Molecular analysis of introgression lines from Cucumis hystrix Chakr. to C. sativus L. Sci. Hortic. 119, 232-235 (2009).

12. Delannay, I. Y. et al. Backcross Introgression of the Cucumis hystrix Genome Increases Genetic Diversity in U.S. Processing Cucumber. J. Am. Soc. Hortic. Sci. 135, 351-361 (2010).

13. Chen, F. et al. The Sequenced Angiosperm Genomes and Genome Databases. Front Plant Sci. 9, 418 (2018).

14. Huang, S. et al. The genome of the cucumber, Cucumis sativus L. Nat. Genet. 41, 1275-1281 (2009).

15. Garcia-Mas, J. et al. The genome of melon (Cucumis melo L.). Proc. Natl Acad. Sci. USA. 109, 11872-11877 (2012).

16. Zhuang, F. et al. Taxonomic relationships of a rare Cucumis species (C. hystrix Chakr.) and its interspecific hybrid with cucumber. Hortscience 41, 571-574 (2006).

17. Ghebretinsae, A. G. et al. Relationships of cucumbers and melons unraveled: Molecular phylogenetics of Cucumis and related genera (Benincaseae, Cucurbitaceae). Am. J. Bot. 94, 1256-1266 (2007). 
18. Renner, S. S. et al. Phylogenetics of Cucumis (Cucurbitaceae): Cucumber (C. sativus) belongs in an Asian/Australian clade far from melon (C. melo). BMC Evol. Biol. 7, 58 (2007).

19. Sebastian, P. et al. Cucumber (Cucumis sativus) and melon (C. melo) have numerous wild relatives in Asia and Australia, and the sister species of melon is from Australia. Proc. Natl Acad. Sci. USA 107, 14269-14273 (2010).

20. Zhang, Y. et al. Chromosomal structures and repetitive sequences divergence in Cucumis species revealed by comparative cytogenetic mapping. BMC Genomics 16, 730 (2015).

21. Han, Y. et al. Chromosome-specific painting in Cucumis Species using bulked oligonucleotides. Genetics 200, 771-779 (2015)

22. Li, D. et al. Syntenic relationships between cucumber (Cucumis sativus L.) and melon (C. melo L.) chromosomes as revealed by comparative genetic mapping. BMC Genomics 12, 396 (2011).

23. Wendel, J. F. \& Doyle, J. J. in Molecular Systematics of Plants II: DNA Sequencing (eds Soltis, D. E., Soltis, P. S. \& Doyle, J. J.) 265-296 (Springer US, 1998).

24. Rokas, A. et al. Genome-scale approaches to resolving incongruence in molecular phylogenies. Nature 425, 798-804 (2003).

25. Nakhleh, L. Computational approaches to species phylogeny inference and gene tree reconciliation. Trends Ecol. Evol. 28, 719-728 (2013).

26. Som, A. Causes, consequences and solutions of phylogenetic incongruence. Brief. Bioinform. 16, 536-548 (2015).

27. Yang, L. et al. Next-generation sequencing, FISH mapping and synteny-based modeling reveal mechanisms of decreasing dysploidy in Cucumis. Plant J. 77 16-30 (2014).

28. Weisenfeld, N. I. et al. Direct determination of diploid genome sequences. Genome Res. 27, 757-767 (2017).

29. Simão, F. A. et al. BUSCO: Assessing genome assembly and annotation completeness with single-copy orthologs. Bioinformatics 31, 3210-3212 (2015).

30. Wang, Y. et al. MCScanX: a toolkit for detection and evolutionary analysis of gene synteny and collinearity. Nucleic Acids Res. 40, e49 (2012).

31. Emms, D. M. \& Kelly, S. OrthoFinder: solving fundamental biases in whole genome comparisons dramatically improves orthogroup inference accuracy. Genome Biol. 16, 157 (2015).

32. Thornton, J. W. \& DeSalle, R. Gene family evolution and homology: genomics meets phylogenetics. Annu. Rev. Genomics Hum. Genet. 1, 41-73 (2000).

33. Demuth, J. P. \& Hahn, M. W. The life and death of gene families. BioEssays N. Rev. Mol. Cell. Dev. Biol. 31, 29-39 (2009).

34. Guo, Y.-L. Gene family evolution in green plants with emphasis on the origination and evolution of Arabidopsis thaliana genes. Plant J. Cell Mol. Biol. 73 941-951 (2013).

35. Sahm, A. et al. PosiGene: automated and easy-to-use pipeline for genome-wide detection of positively selected genes. Nucleic Acids Res. 45, e100 (2017).

36. Kandoth, P. K. et al. The soybean Rhg1 locus for resistance to the soybean cyst nematode Heterodera glycines regulates the expression of a large number of stress- and defense-related genes in degenerating feeding cells. Plant Physiol. 155, 1960-1975 (2011)

37. Jin, J. et al. Arabidopsis peroxidase AtPRX53 influences cell elongation and susceptibility to Heterodera schachtii. Plant Signal. Behav. 6, 1778-1786 (2011).

38. Dhar, N. et al. An Arabidopsis DISEASE RELATED NONSPECIFIC LIPID TRANSFER PROTEIN 1 is required for resistance against various phytopathogens and tolerance to salt stress. Gene $\mathbf{7 5 3}, 144802$ (2020).

39. Kourelis, J. \& van der Hoorn, R. A. L. Defended to the nines: 25 years of resistance gene cloning identifies nine mechanisms for $\mathrm{R}$ protein function. Plant Cell 30, 285-299 (2018).

40. Li, P. et al. RGAugury: a pipeline for genome-wide prediction of resistance gene analogs (RGAs) in plants. BMC Genomics 17, 852 (2016).

41. McHale, L. et al. Plant NBS-LRR proteins: adaptable guards. Genome Biol. 7, 212 (2006).

42. Marone, D. et al. Plant nucleotide binding site-leucine-rich repeat (NBS-LRR) genes: active guardians in host defense responses. Int. J. Mol. Sci. 14, 7302-7326 (2013).

43. Meyers, B. C. et al. Genome-wide analysis of NBS-LRR-encoding genes in Arabidopsis. Plant Cell 15, 809-834 (2003).

44. Monosi, B. et al. Full-genome analysis of resistance gene homologues in rice. Theor. Appl. Genet. Theor. Angew. Genet. 109, 1434-1447 (2004).

45. Leister, D. Tandem and segmental gene duplication and recombination in the evolution of plant disease resistance gene. Trends Genet. 20, 116-122 (2004).
46. Singh, A. K. \& Yadava, K. S. An analysis of interspecific hybrids and phylogenetic implications in Cucumis (Cucurbitaceae). Plant Syst. Evol. 147, 237-252 (1984).

47. Naranjo, T. The use of homoeologous pairing in the identification of homoeologous relationships in Triticeae. Hereditas 116, 219-223 (1992).

48. Rieseberg, L. H. Chromosomal rearrangements and speciation. Trends Ecol. Evol. 16, 351-358 (2001).

49. Meyer, R. S. \& Purugganan, M. D. Evolution of crop species: genetics of domestication and diversification. Nat. Rev. Genet. 14, 840-852 (2013).

50. Zhao, G. et al. A comprehensive genome variation map of melon identifies multiple domestication events and loci influencing agronomic traits. Nat Genet. 51, 1607-1615 (2019).

51. Varshney, R. K. et al. Harvesting the promising fruits of genomics: applying genome sequencing technologies to crop breeding. PLOS Biol. 12, e1001883 (2014).

52. Crossa, J. et al. Genomic selection in plant breeding: methods, models, and perspectives. Trends Plant Sci. 22, 961-975 (2017).

53. Zhong, S. et al. High-throughput Illumina strand-specific RNA sequencing library preparation. Cold Spring Harb. Protoc. 2011, 940-949 (2011).

54. Liu, B. et al. Estimation of genomic characteristics by analyzing k-mer frequency in de novo genome projects. Preprint at https:/arxiv.org/abs/ 1308.2012 (2020).

55. Walker, B. J. et al. Pilon: an integrated tool for comprehensive microbial variant detection and genome assembly improvement. PloS One 9, e112963 (2014).

56. Chen, S. et al. fastp: an ultra-fast all-in-one FASTQ preprocessor. Bioinforma. Oxf. Engl. 34, i884-i890 (2018).

57. Zimin, A. V. et al. The MaSuRCA genome assembler. Bioinformatics 29, 2669-2677 (2013)

58. Koren, S. et al. Canu: scalable and accurate long-read assembly via adaptive kmer weighting and repeat separation. Genome Res 27, 722-736 (2017).

59. English, A. C. et al. Mind the gap: upgrading genomes with Pacific Biosciences RS long-read sequencing technology. PloS One 7, e47768 (2012).

60. Boetzer, M. et al. Scaffolding pre-assembled contigs using SSPACE. Bioinforma. Oxf. Engl. 27, 578-579 (2011)

61. Leggett, R. M. et al. NextClip: an analysis and read preparation tool for Nextera Long Mate Pair libraries. Bioinformatics 30, 566-568 (2014).

62. Schuler, G. D. Sequence mapping by electronic PCR. Genome Res 7, 541-550 (1997).

63. Tang, $H$. et al. ALLMAPS: robust scaffold ordering based on multiple maps. Genome Biol. 16, 3 (2015).

64. Li, H. \& Durbin, R. Fast and accurate short read alignment with BurrowsWheeler transform. Bioinforma. Oxf. Engl. 25, 1754-1760 (2009).

65. Kim, D. et al. Graph-based genome alignment and genotyping with HISAT2 and HISAT-genotype. Nat. Biotechnol. 37, 907-915 (2019).

66. Pertea, M. et al. StringTie enables improved reconstruction of a transcriptome from RNA-seq reads. Nat. Biotechnol. 33, 290-295 (2015).

67. Majoros, W. H. et al. TigrScan and GlimmerHMM: two open source ab initio eukaryotic gene-finders. Bioinformatics 20, 2878-2879 (2004).

68. Hoff, K. J. \& Stanke, M. Predicting genes in single genomes with AUGUSTUS. Curr. Protoc. Bioinforma. 65, e57 (2019).

69. Korf, I. Gene finding in novel genomes. BMC Bioinforma. 5, 59 (2004).

70. Birney, E. GeneWise and genomewise. Genome Res 14, 988-995 (2004).

71. Haas, B. J. et al. Automated eukaryotic gene structure annotation using EVidenceModeler and the program to assemble spliced alignments. Genome Biol. 9. R7 (2008).

72. Marçais, G. et al. MUMmer4: a fast and versatile genome alignment system PLoS Comput. Biol. 14, e1005944 (2018).

73. Cock, P. J. A. et al. NCBI BLAST+ integrated into Galaxy. GigaScience 4, 39 (2015).

74. Zhang, Z. et al. ParaAT: a parallel tool for constructing multiple protein-coding DNA alignments. Biochem. Biophys. Res. Commun. 419, 779-781 (2012).

75. Zhang, Z. et al. KaKs_Calculator: calculating Ka and Ks through model selection and model averaging. GPB 4, 259-263 (2006).

76. De Bie, T. et al. CAFE: a computational tool for the study of gene family evolution. Bioinformatics 22, 1269-1271 (2006).

77. Stamatakis, A. RAxML version 8: a tool for phylogenetic analysis and postanalysis of large phylogenies. Bioinformatics 30, 1312-1313 (2014).

78. Yang, Z. PAML 4: phylogenetic analysis by maximum likelihood. Mol. Biol. Evol. 24, 1586-1591 (2007)

79. Edgar, R. C. MUSCLE: multiple sequence alignment with high accuracy and high throughput. Nucleic Acids Res. 32, 1792-1797 (2004). 
80. Nguyen, L.-T. et al. IQ-TREE: a fast and effective stochastic algorithm for estimating maximum-likelihood phylogenies. Mol. Biol. Evol. 32, 268-274 (2015).

81. Letunic, I. \& Bork, P. Interactive Tree Of Life (iTOL) v4: Recent updates and new developments. Nucleic Acids Res. 47, W256-W259 (2019).

82. Wu, H. et al. A high-quality sponge gourd (Luffa cylindrica) genome. Hortic. Res. 7, 1-10 (2020)

83. Cui, J. et al. Whole-genome sequencing provides insights into the genetic diversity and domestication of bitter gourd (Momordica spp.). Hortic. Res. 7 $1-11$ (2020).
84. Zhao, Q. et al. Oligo-painting and GISH reveal meiotic chromosome biases and increased meiotic stability in synthetic allotetraploid Cucumis $\times$ hytivus with dysploid parental karyotypes. BMC Plant Biol. 19, 471 (2019).

85. Wang, Y. et al. Identification of all homoeologous chromosomes of newly synthetic allotetraploid Cucumis $\times$ hytivus and its wild parent reveals stable subgenome structure. Chromosoma 126, 713-728 (2017)

86. Bi, Y. et al. Flexible chromosome painting based on multiplex PCR of oligonucleotides and its application for comparative chromosome analyses in Cucumis. Plant J. 102, 178-186 (2020). 